\title{
Measurements of magnetic fields over the pulsation cycle in six roAp stars with FORS 1 at the VLT`
}

\author{
S. Hubrig ${ }^{1}$, D. W. Kurtz ${ }^{2}$, S. Bagnulo ${ }^{1}$, T. Szeifert ${ }^{1}$, M. Schöller ${ }^{1}$, G. Mathys ${ }^{1}$, and W. A. Dziembowski ${ }^{3}$ \\ 1 European Southern Observatory, Casilla 19001, Santiago 19, Chile \\ 2 Centre for Astrophysics, University of Central Lancashire, Preston, PR1 2HE, UK \\ 3 Warsaw University Observatory, Al. Ujazdowskie, 4, 00-478 Warsawa, Poland
}

Received 12 June 2003 / Accepted 29 August 2003

\begin{abstract}
With FORS 1 at the VLT we have tried for the first time to measure the magnetic field variation over the pulsation cycle in six roAp stars to begin the study of how the magnetic field and pulsation interact. For the star HD 101065, which has one of the highest photometric pulsation amplitudes of any roAp star, we found a signal at the known photometric pulsation frequency at the $3 \sigma$ level in one data set; however this could not be confirmed by later observations. A preliminary simple calculation of the expected magnetic variations over the pulsation cycle suggests that they are of the same order as our current noise levels, leading us to expect that further observations with increased $\mathrm{S} / \mathrm{N}$ have a good chance of achieving an unequivocal detection.
\end{abstract}

Key words. stars: magnetic fields - stars: oscillations - stars: chemically peculiar

\section{Introduction}

The rapidly oscillating stars (roAp) were the first stars for which solar-like high-overtone $p$-mode pulsations have been definitely detected and are, therefore, prime candidates for asteroseismological studies. The roAp stars are cool chemically peculiar stars that pulsate in high-overtone $(n \gg l)$, low-degree $(l \leq 3) p$-modes with periods from about 6 to $15 \mathrm{~min}$ and typical photometric $B$ amplitudes of a few mmag. There are 32 such stars currently known. Detailed reviews of the roAp stars have been published by Kurtz (1990), Matthews (1991), and Kurtz \& Martinez (2000).

The pulsations of roAp stars are to a large extent governed by their magnetic field (Bigot et al. 2000; Cunha \& Gough 2000). An effect of magnetic field on $p$-mode oscillations has been recently considered by Bigot \& Dziembowski (2002). Their theory of the interaction of rotation, pulsation and the magnetic field suggests a new model, the improved oblique pulsator model, that is a significant departure from the standard oblique pulsator model for roAp stars. They suggest that the light variations are caused by a pulsation mode in which the stellar surface moves in a plane that is inclined to both the rotation and the magnetic axes of the star. The displacement vector describes an ellipse in that plane with the pulsation period, and

Send offprint requests to: S. Hubrig, e-mail: shubrig@eso.org

* Based on observations obtained at the European Southern Observatory, Paranal, Chile (ESO programmes Nos. 69.D-0210 and 270.D-5023). the whole pattern rotates with the rotation of the oblique magnetic field.

From the theoretical considerations, a simple estimate shows that a pulsationally-modulated variation of the order of $\approx 10^{2} \mathrm{G}$ may exist in the outer atmospheric layers of roAp stars with $\mathrm{kG}$ magnetic fields. Assuming that the unperturbed field is nearly force-free and ignoring the angular derivatives of the displacement vector $\boldsymbol{\xi}$, we have a good approximation for the magnetic field amplitude

$\delta B_{\theta}=B_{r} \frac{\partial \xi_{\theta}}{\partial r}-B_{\theta} \frac{\partial \xi_{r}}{\partial r}$

and

$\left|\delta B_{r}\right| \ll\left|\delta B_{\theta}\right|$.

The local plane-parallel approximation for $\boldsymbol{\xi}$ is well justified in our case of high-frequency low-degree modes in outer layers. In the same approximation we have for the perturbed density

$\frac{\delta \rho}{\rho}=-\frac{\partial \xi_{r}}{\partial r}$

From these assumptions we get the following crude estimate:

$\left|\frac{\delta B_{\theta}}{B}\right| \sim\left|\frac{\delta \rho}{\rho}\right|$.

Both numerical nonadiabatic calculations of oscillations in nonmagnetic stars and the quasi-adiabatic approximation suggest that the magnitude of the relative intensity amplitude 
should be of the same order as that of the density. If we assume the same for magnetic stars, which seems reasonable, then a $10 \mathrm{mmag}$ in light amplitude translates to $1 \%$ variations of the field. This number refers to the subphotosperic layer where the light amplitude is fixed. An estimate for the expected field variations in the atmosphere may be obtained only with radial velocity data. For example, from the amplitude rise of $\delta V \sim 0.8 \mathrm{~km} \mathrm{~s}^{-1}$ in the atmosphere $(h \sim 1000 \mathrm{~km})$ we would get

$\left|\frac{\partial \xi}{\partial r}\right| \sim \frac{P \delta V}{2 h \pi} \sim 0.08$

where $P$ is the pulsation period. Considering that non-adiabatic oscillations in magnetic stars have not been modelled, our calculation of the possible pulsationally-modulated variation of the magnetic field is simply a first order-of-magnitude estimate of the effect that may be expected. The adopted radial velocity amplitude rise of $\sim 0.8 \mathrm{~km} \mathrm{~s}^{-1}$ is based on the radial velocity measurements of the Pr III line at $\lambda 6160 \AA$ in $\gamma$ Equ (Kochukhov \& Ryabchikova 2001a). This estimate implies magnetic field variations at the level of $8 \%$. In general, a large pulsational amplitude is found in doubly ionized rare-earth ions, although a large pulsational amplitude in the $\mathrm{H}_{\alpha}$ line, up to $1.46 \mathrm{~km} \mathrm{~s}^{-1}$, has also been reported for the roAp star HD 83368 (Balona 2002). The pulsational velocity amplitudes in the $\mathrm{H}_{\beta}$ and $\mathrm{H}_{\gamma}$ lines in the spectra of this star show, however, much lower amplitudes. For the star HD 128898, Kochukhov \& Ryabchikova (2001b) detected modulation of the radial velocity amplitude in the $\mathrm{Nd}$ III line at $\lambda 6145 \AA$ with a variation between 300 and $500 \mathrm{~m} \mathrm{~s}^{-1}$. The pulsations in the radial velocities of the rare-earth lines in this star have been confirmed by Balona \& Zima (2002), but they were only very weakly present in the radial velocities of the hydrogen lines. An analysis of individual spectral lines in the roAp star HD 137949 revealed the pulsational radial velocity amplitude ranging from $320 \mathrm{~m} \mathrm{~s}^{-1}$ to an amplitude as low as $7 \mathrm{~m} \mathrm{~s}^{-1}$ (Hatzes et al. 1999). To summarize, the available radial velocity data imply an estimate for the expected magnetic field variations in the atmospheres of roAp stars at the level of 1 to $14 \%$.

This estimate is given here as an illustration of the fact that some theoretical arguments suggest that pulsation may induce magnetic field variations that will be detectable through observation with currently existing instruments. This represents the motivation underlying the present study. Admittedly, ambiguities and unknowns remain in our current understanding of the physics of pulsation in roAp stars, and possible alternative theoretical interpretations may lead to somewhat different estimates of the magnetic field variations to be expected - some possibly below the detection threshold. Accordingly, observations of magnetic field variations may provide a useful discriminant between various models that are currently possible.

Despite of the importance of magnetic fields for the proper understanding of pulsational properties of roAp stars, these fields have scarcely been studied until now (Mathys 2003; Hubrig et al. 2004). How this pulsation pattern interacts with the magnetic field on the time-scale of the pulsations was entirely untested observationally before our VLT runs in April and September 2002. The primary purpose of this paper is to provide observational constraints on such magnetic field variations. Because of the short duration of the pulsations $(6-15 \mathrm{~min})$, such observations could not be obtained in the past with $4-\mathrm{m}$ class telescopes. The required integration time for measuring the magnetic field in a $V=8 \mathrm{mag}$ star with UT1 and FORS1 is only a few tens of seconds. This has allowed us to carry out an entirely new kind of observation to search for magnetic variability over the pulsation cycle.

Here we present new results of measurements of the mean longitudinal field of six roAp stars obtained from low resolution spectropolarimetry with FORS 1 at the VLT.

\section{Observations and data reduction}

Time series of spectra of bright roAp stars were obtained with FORS 1 (FOcal Reducer low dispersion Spectrograph) mounted on the 8-m Melipal (UT3) telescope of the VLT in April 2002 (HD 83368, HD 101065, HD 128898 and HD 137949) and in September 2002 (HD 201601 and HD 217522). The FORS 1 multi-mode instrument is equipped with polarization analyzing optics comprising superachromatic half-wave and quarter-wave phase retarder plates, and a Wollaston prism with a beam divergence of $22^{\prime \prime}$ in standard resolution mode (Appenzeller et al. 1998). During our April run we used the GRISM 600B to cover all $\mathrm{H}$ Balmer lines from $\mathrm{H}_{\beta}$ to the Balmer jump, and during the September run we observed with GRISM600R in the region which includes $\mathrm{H}_{\alpha}$ and $\mathrm{H}_{\beta}$, from 4770 to $6900 \AA$. Both grisms have 600 grooves $\mathrm{mm}^{-1}$; with the narrowest available slit width of 0.4 they give a spectral resolving power of $R \sim 2000$ and $R \sim 2900$, respectively. Wavelength calibrations were taken during day time for the two different retarder waveplate setups $\left(\alpha=+45^{\circ}\right.$ and $\left.-45^{\circ}\right)$ which are the same as those used for the observations. Wavelength calibration was performed by associating with each individual science spectrum the calibration frame obtained with the similar orientation of the retarder waveplate. Ordinary and extraordinary beams were independently calibrated with the corresponding beams of the reference spectrum. As has been previously shown by Landstreet (1982), in the weak field regime, the mean longitudinal field can be derived from the difference between the circular polarizations observed in the red and blue wings of the hydrogen line profiles using the formula

$\frac{V}{I}=-\frac{g_{\mathrm{eff}} e \lambda^{2}}{4 \pi m_{\mathrm{e}} c^{2}} \frac{1}{I} \frac{\mathrm{d} I}{\mathrm{~d} \lambda}\left\langle\mathcal{B}_{z}\right\rangle$,

where $V$ is the Stokes parameter which measures the circular polarization, $I$ is the intensity in the unpolarized spectrum, $g_{\text {eff }}$ is the effective Lande factor, $e$ is the electron charge, $\lambda$ is the wavelength, $m_{\mathrm{e}}$ the electron mass, $c$ the speed of light, and $\left\langle\mathcal{B}_{z}\right\rangle$ is the mean longitudinal field. To minimize the cross-talk effect we executed the sequence $+45-45,-45+45,+45-45$ etc. and calculated the values $V / I$ using:

$\frac{V}{I}=\frac{1}{2}\left\{\left(\frac{f^{\mathrm{o}}-f^{\mathrm{e}}}{f^{\mathrm{o}}+f^{\mathrm{e}}}\right)_{\alpha=-45^{\circ}}-\left(\frac{f^{\mathrm{o}}-f^{\mathrm{e}}}{f^{\mathrm{o}}+f^{\mathrm{e}}}\right)_{\alpha=+45^{\circ}}\right\}$,

where $\alpha$ gives the position angle of the retarder waveplate and $f^{\circ}$ and $f^{\mathrm{e}}$ are ordinary and extraordinary beams, respectively. In our calculations we assumed a Landé factor $g_{\text {eff }}=1$ 
Table 1. Wavelength ranges around the hydrogen Balmer lines for which the Landé factor has been set to 1 . In all other wavelength ranges the Landé factor $g_{\text {eff }}=1.25$ has been adopted.

\begin{tabular}{cc}
\hline \hline Line(s) & Wavelength range $[\AA]$ \\
\hline $\mathrm{H}_{16}-\mathrm{H}_{10}$ & $3701-3801$ \\
$\mathrm{H}_{9}$ & $3820.5-3852.5$ \\
$\mathrm{H}_{8}$ & $3870.2-3910.2$ \\
$\mathrm{H}_{\epsilon}$ & $3941.2-4001.2$ \\
$\mathrm{H}_{\delta}$ & $4082.9-4122.9$ \\
$\mathrm{H}_{\gamma}$ & $4311.7-4371.7$ \\
$\mathrm{H}_{\beta}$ & $4812.7-4912.7$ \\
$\mathrm{H}_{\alpha}$ & $6512-6612$ \\
\hline
\end{tabular}

for hydrogen lines and $g_{\mathrm{eff}}=1.25$ for metal lines. Table 1 lists the wavelength ranges corresponding to the hydrogen Balmer lines for which the Landé factor has been set to 1. Furthermore, in our reduction procedure the spectral regions containing telluric lines have been excluded in the measurements of magnetic field. More details of the observing technique are given by Bagnulo et al. (2002) and Hubrig et al. (2003). The errors of the measurements of the polarization have been determined from photon counting statistics and have been converted to errors of field measurements. On each night an additional star with a well-defined strong longitudinal field was selected to check that the instrument was functioning properly. The star HD 94660, which was observed in April, has a longitudinal magnetic field that varies about a mean value of $\sim-1900 \mathrm{G}$ with a low amplitude $(\sim 160 \mathrm{G}$ peak-to-peak) over a period of $2800 \mathrm{~d}$ (Mathys et al. 2003). The result of our measurement using the whole spectral region from 3500 to $5800 \AA,\left\langle\mathcal{B}_{z}\right\rangle=-2056 \pm 19 \mathrm{G}$, is fully consistent with the value of the longitudinal field at the considered rotation phase that is expected from the variation curve defined by Mathys et al. (2003). The measurements obtained from the individual Balmer lines show slightly different results. For instance, using for the measurements exclusively the $\mathrm{H}_{\beta}$ line we obtain $\left\langle\mathcal{B}_{z}\right\rangle_{\mathrm{H}_{\beta}}=-2442 \pm 73 \mathrm{G}$. The reasons for different values of $\left\langle\mathcal{B}_{z}\right\rangle$ derived from Balmer lines in comparison to the values obtained using the whole region are discussed in more detail by Bagnulo et al. (2002). At this point we would like to mention that for all stars of our sample the magnetic field measured in the wings of $\mathrm{H}_{\beta}$ is systematically larger, up to $500 \mathrm{G}$, than the field derived from the $\mathrm{H}_{\gamma}, \mathrm{H}_{\delta}$ and $\mathrm{H}_{\epsilon}$ lines. The inspection of the behaviour of the polarized spectrum in the wings of the $\mathrm{H}_{\beta}$ line reveals a very strong signal at the wavelength $\lambda \approx 4876 \AA$ where the blend of strong lines belonging to $\mathrm{Nd}$ II and $\mathrm{Cr}$ II is located (Fig. 1). This feature probably accounts for the excess of magnetic field measured in the region of $\mathrm{H}_{\beta}$. However, high resolution spectropolarimetric observations of these stars are needed to verify the origin of such a strong polarization signal.

The star HD 187474, which has a rotation period of $6.4 \mathrm{yr}$ and a longitudinal magnetic field ranging from $\approx-2 \mathrm{kG}$ to $2 \mathrm{kG}$, was observed during the night of 14 September 2002 at the

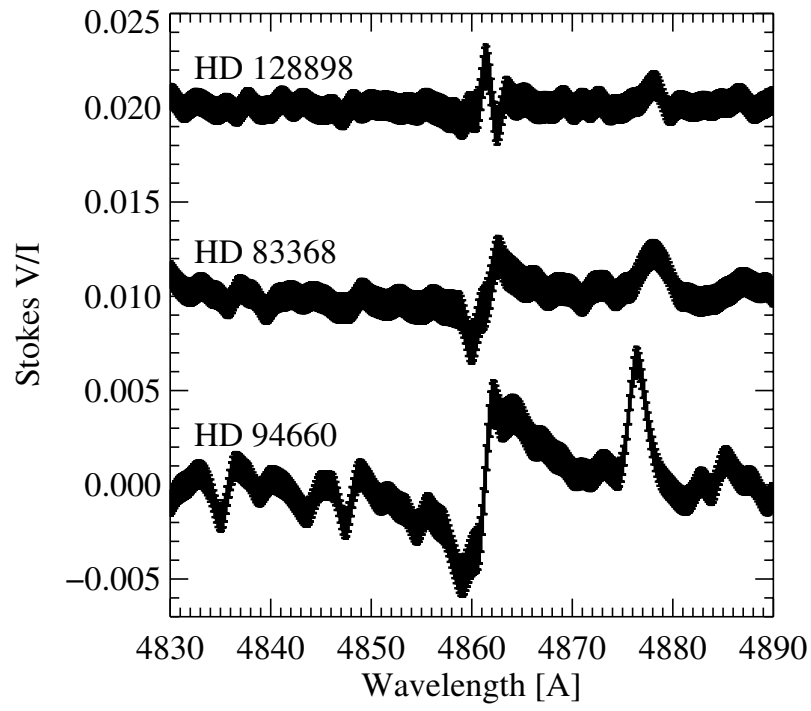

Fig. 1. Stokes $V$ profiles in the region of the $\mathrm{H}_{\beta}$ line in the two roAp stars HD 128898 and HD 83368 and in the strongly magnetic Ap star HD 94660. The spectra of HD 83368 and HD 128898 are shifted in the vertical direction by 0.01 and 0.02 respectively. The thickness of the plotted lines corresponds to the uncertainty of the measurement of polarization determined from photon noise. A strong polarization signal close to the wavelength $\lambda \approx 4876 \AA$ is probably due to the presence of the blend of strong lines belonging to Nd II and Cr II.

rotation phase 0.46 . The derived value of the magnetic field, $\left\langle\mathcal{B}_{z}\right\rangle=-1849 \pm 47 \mathrm{G}$, fits very well to the observations at the same phase presented by Mathys et al. (1997).

The individual measurements of the longitudinal magnetic field for each star are presented in Tables 2 to 7 . After finishing the time series for each star in our sample of four stars in the observing night in April we decided to use the GRISM 600R for a few exposures to check the consistency of the magnetic field measurements in the region including the $\mathrm{H}_{\alpha}$ line with that which contains all other Balmer lines. No systematic difference has been found between the measurements in the two different spectral regions.

\section{Results for individual stars}

For each roAp star we took a continuous series of sets of 2 exposures (with the retarder waveplate oriented at different angles), distributed along many consecutive pulsation cycles. As the observed spectral range includes all Balmer lines from $\mathrm{H}_{\beta}$ bluewards, the analysis of their Stokes $V$ profiles observed in each exposure permits us to obtain an estimate of the mean longitudinal field with an accuracy better than $100 \mathrm{G}$ with a few seconds of exposure time for HD 128898, which is the brightest star in our sample, and about $60 \mathrm{~s}$ for the faintest star HD 101065. Among the roAp stars, the selected targets have pulsation periods from 6.8 to $13.9 \mathrm{~min}$. Since the exposure time is only a fraction of the pulsation period for all considered stars, by Fourier analysing our results over many cycles of pulsation we have been able to examine whether there exist variations of the order of a few $100 \mathrm{G}$ over the pulsation period. Pulsational variability of Stokes $V$ during four consecutive 
Table 2. Mean longitudinal magnetic field for the time series of HD 83368.

\begin{tabular}{ccc|ccc}
\hline \hline MJD & $\left\langle\mathcal{B}_{z}\right\rangle$ & $\sigma_{z}$ & MJD & $\left\langle\mathcal{B}_{z}\right\rangle$ & $\sigma_{z}$ \\
& {$[\mathrm{G}]$} & {$[\mathrm{G}]$} & & {$[\mathrm{G}]$} & {$[\mathrm{G}]$} \\
\hline 52382.977731 & -868 & 60 & 52383.039369 & -786 & 54 \\
52382.979433 & -861 & 60 & 52383.041174 & -854 & 57 \\
52382.981137 & -805 & 60 & 52383.042984 & -818 & 60 \\
52382.982841 & -608 & 60 & 52383.044806 & -829 & 60 \\
52382.984553 & -1057 & 63 & 52383.046728 & -879 & 47 \\
52382.986283 & -788 & 57 & 52383.048638 & -720 & 51 \\
52382.988050 & -777 & 54 & 52383.050505 & -830 & 54 \\
52382.989816 & -783 & 60 & 52383.052323 & -990 & 57 \\
52382.991590 & -879 & 60 & 52383.054130 & -718 & 60 \\
52382.993386 & -829 & 54 & 52383.055971 & -681 & 54 \\
52382.995213 & -839 & 51 & 52383.057846 & -920 & 51 \\
52382.997032 & -845 & 54 & 52383.059721 & -833 & 54 \\
52382.998804 & -892 & 57 & 52383.061596 & -932 & 54 \\
52383.000575 & -771 & 57 & 52383.063473 & -764 & 51 \\
52383.002350 & -782 & 57 & 52383.065349 & -821 & 51 \\
52383.006425 & -1181 & 54 & 52383.069270 & -805 & 54 \\
52383.008209 & -905 & 57 & 52383.071105 & -848 & 51 \\
52383.009994 & -830 & 54 & 52383.072939 & -910 & 51 \\
52383.011779 & -575 & 57 & 52383.074751 & -790 & 57 \\
52383.013568 & -897 & 54 & 52383.076566 & -965 & 51 \\
52383.015354 & -1115 & 54 & 52383.078386 & -864 & 51 \\
52383.017147 & -767 & 54 & 52383.080183 & -1011 & 57 \\
52383.018943 & -940 & 57 & 52383.081960 & -896 & 57 \\
52383.020728 & -820 & 60 & 52383.083742 & -817 & 57 \\
52383.022515 & -957 & 60 & 52383.085520 & -920 & 57 \\
52383.024320 & -834 & 60 & 52383.087300 & -801 & 57 \\
52383.026218 & -955 & 51 & 52383.089080 & -868 & 60 \\
52383.028119 & -687 & 54 & 52383.090877 & -812 & 60 \\
52383.029930 & -817 & 60 & 52383.092768 & -863 & 57 \\
52383.031730 & -950 & 54 & 52383.094707 & -635 & 51 \\
\hline & & & & &
\end{tabular}

exposures are presented in Fig. 2 for the star HD 128898, which has the smallest measured longitudinal field, and in Fig. 3 for the star HD 137949, which has the largest measured longitudinal field.

Given the low resolution of FORS 1, metallic lines in the spectra of roAp stars appear mostly as unresolved blends. Using observations of the strongly magnetic star HD 94660, Bagnulo et al. (2002) studied the impact of metallic line blends on the determination of the longitudinal field in comparison to measurements of the magnetic field restricted exclusively to the wavelength region containing hydrogen Balmer lines. They showed that the determination of the longitudinal field using the full wavelength range, including all metallic lines, is reasonably consistent with that obtained within wavelength windows around individual Balmer lines. The roAp stars in our
Table 3. Mean longitudinal magnetic field for the time series of HD 101065 obtained in April 2002.

\begin{tabular}{ccc|ccc}
\hline \hline MJD & $\left\langle\mathcal{B}_{z}\right\rangle$ & $\sigma_{z}$ & MJD & $\left\langle\mathcal{B}_{z}\right\rangle$ & $\sigma_{z}$ \\
& {$[\mathrm{G}]$} & {$[\mathrm{G}]$} & & {$[\mathrm{G}]$} & {$[\mathrm{G}]$} \\
\hline 52383.148501 & -1061 & 51 & 52383.202286 & -914 & 47 \\
52383.151370 & -1072 & 51 & 52383.205434 & -1032 & 51 \\
52383.154296 & -1075 & 51 & 52383.208556 & -949 & 51 \\
52383.157568 & -968 & 44 & 52383.211683 & -1023 & 51 \\
52383.160875 & -1105 & 44 & 52383.214809 & -1015 & 54 \\
52383.164007 & -1128 & 51 & 52383.217932 & -1068 & 54 \\
52383.167110 & -926 & 51 & 52383.221058 & -1021 & 54 \\
52383.170237 & -1075 & 51 & 52383.224218 & -1043 & 57 \\
52383.173541 & -1025 & 51 & 52383.227554 & -1075 & 51 \\
52383.176883 & -1072 & 54 & 52383.230917 & -1119 & 51 \\
52383.180222 & -1060 & 54 & 52383.234282 & -1019 & 54 \\
52383.183554 & -999 & 51 & 52383.237652 & -1085 & 54 \\
52383.186888 & -1105 & 51 & 52383.241020 & -999 & 57 \\
52383.190224 & -1052 & 47 & 52383.244393 & -1017 & 60 \\
52383.193564 & -1063 & 47 & 52383.247762 & -1062 & 60 \\
\hline
\end{tabular}

Table 4. Mean longitudinal magnetic field for the time series of HD 128898 .

\begin{tabular}{crr|rrr}
\hline \hline MJD & $\left\langle\mathcal{B}_{z}\right\rangle$ & $\sigma_{z}$ & MJD & \multicolumn{1}{c}{$\left\langle\mathcal{B}_{z}\right\rangle$} & $\sigma_{z}$ \\
& {$[\mathrm{G}]$} & {$[\mathrm{G}]$} & & {$[\mathrm{G}]$} & {$[\mathrm{G}]$} \\
\hline 52383.278303 & -5 & 60 & 52383.301002 & -306 & 63 \\
52383.279870 & -361 & 60 & 52383.302584 & -1 & 66 \\
52383.281422 & -63 & 63 & 52383.304163 & -195 & 70 \\
52383.282986 & -368 & 63 & 52383.305740 & -385 & 70 \\
52383.284544 & -291 & 63 & 52383.307315 & -287 & 70 \\
52383.288427 & -163 & 60 & 52383.308895 & -342 & 63 \\
52383.289992 & -256 & 63 & 52383.310474 & -233 & 70 \\
52383.291561 & -478 & 63 & 52383.314201 & -230 & 57 \\
52383.293135 & -472 & 63 & 52383.315821 & -148 & 54 \\
52383.294707 & -298 & 60 & 52383.317441 & -251 & 57 \\
52383.296279 & -321 & 63 & 52383.319067 & -23 & 60 \\
52383.297857 & -40 & 63 & 52383.320686 & -310 & 57 \\
52383.299426 & -118 & 63 & 52383.322304 & -271 & 57 \\
\hline
\end{tabular}

sample are much cooler than HD 94660 which has the spectral type A0, and their spectra are incomparably line rich. In addition, spectral variability throughout the pulsation cycle has been found in some roAp stars. For example, Kochukhov \& Ryabchikova (2001a) have shown that in $\gamma$ Equ the spectral lines of Pr III and Nd III show significant radial velocity variations with the pulsation period, while other lines in the spectrum show none. Balona (2002) and Balona \& Zima (2002) get similar results for HD 83368 and HD 24712, respectively. Interestingly, they have good evidence that the amplitude of the pulsation is higher in $\mathrm{H}_{\alpha}$ than in $\mathrm{H}_{\beta}$ and $\mathrm{H}_{\gamma}$ in those stars. 
Table 5. Mean longitudinal magnetic field for the time series of HD 137949.

\begin{tabular}{ccc|ccc}
\hline \hline MJD & $\left\langle\mathcal{B}_{z}\right\rangle$ & \multicolumn{1}{c}{$\sigma_{z}$} & MJD & $\left\langle\mathcal{B}_{z}\right\rangle$ & $\sigma_{z}$ \\
& {$[\mathrm{G}]$} & {$[\mathrm{G}]$} & & {$[\mathrm{G}]$} & {$[\mathrm{G}]$} \\
\hline 52383.341268 & 2111 & 47 & 52383.371795 & 2193 & 51 \\
52383.343093 & 2161 & 47 & 52383.373714 & 2003 & 54 \\
52383.344925 & 2204 & 51 & 52383.375634 & 2092 & 51 \\
52383.346754 & 2078 & 51 & 52383.377559 & 2279 & 54 \\
52383.348591 & 2154 & 54 & 52383.379490 & 2107 & 54 \\
52383.350428 & 2132 & 51 & 52383.381451 & 2105 & 54 \\
52383.352264 & 2154 & 57 & 52383.383430 & 2108 & 51 \\
52383.354154 & 2121 & 51 & 52383.385404 & 2140 & 57 \\
52383.356050 & 2246 & 51 & 52383.387515 & 2190 & 54 \\
52383.357898 & 2154 & 54 & 52383.389743 & 2164 & 47 \\
52383.359796 & 2159 & 47 & 52383.391962 & 2139 & 47 \\
52383.361710 & 2223 & 47 & 52383.394179 & 2072 & 47 \\
52383.363619 & 2162 & 47 & 52383.396398 & 2168 & 47 \\
52383.365523 & 2138 & 47 & 52383.398609 & 2113 & 47 \\
52383.367434 & 2198 & 51 & 52383.400823 & 2132 & 47 \\
\hline
\end{tabular}

Table 6. Mean longitudinal magnetic field for the time series of HD 201601.

\begin{tabular}{ccc|ccc}
\hline \hline MJD & $\left\langle\mathcal{B}_{z}\right\rangle$ & $\sigma_{z}$ & MJD & $\left\langle\mathcal{B}_{z}\right\rangle$ & $\sigma_{z}$ \\
& {$[\mathrm{G}]$} & {$[\mathrm{G}]$} & & \multicolumn{1}{c}{$[\mathrm{G}]$} & {$[\mathrm{G}]$} \\
\hline 52530.991626 & -1081 & 60 & 52531.044233 & -1086 & 57 \\
52530.995482 & -1135 & 54 & 52531.047133 & -915 & 57 \\
52531.017022 & -842 & 47 & 52531.051108 & -1112 & 54 \\
52531.026988 & -1065 & 57 & 52531.054008 & -1106 & 54 \\
52531.029854 & -739 & 57 & 52531.056909 & -930 & 51 \\
52531.032729 & -1164 & 60 & 52531.059824 & -1084 & 51 \\
52531.035599 & -1157 & 57 & 52531.084795 & -1286 & 79 \\
52531.038471 & -1273 & 51 & 52531.093915 & -777 & 47 \\
52531.041351 & -1163 & 54 & 52531.096943 & -1384 & 51 \\
\hline
\end{tabular}

Knudsen (2000) detected the pulsation modes in equivalent width variations of $\mathrm{H}_{\alpha}$ in the star HD 24712. Bisector measurements of the $\mathrm{H}_{\alpha}$ line of the roAp stars HD 128898 (Baldry et al. 1999) and HD 83368 (Baldry \& Bedding 2000) have provided pulsational depth information for the first time. Finally, Kurtz et al. (2003) have resolved the magneto-acoustic boundary layer as a function of atmospheric depth in HD 166473 using high time resolution VLT UVES spectra. They obtain radial velocity uncertainties of only $2 \mathrm{~m} \mathrm{~s}^{-1}$, and also find no variation in the velocities of $\mathrm{Fe}$ lines with velocities up to $80 \mathrm{~m} \mathrm{~s}^{-1}$ in lines of $\mathrm{Nd}$ and $\mathrm{Pr}$. The derived amplitudes and phases of the radial velocity variations as a function of depth in roAp stars allow detailed atmospheric constraints to be deduced.

Because of the suggestion by Balona (2002) that the pulsation amplitude is lower for $\mathrm{H}_{\beta}$ and $\mathrm{H}_{\gamma}$ than for $\mathrm{H} \alpha$, and because nothing is known about the pulsational behaviour of
Table 7. Mean longitudinal magnetic field for the time series of HD 217522.

\begin{tabular}{|c|c|c|c|c|c|}
\hline MJD & $\begin{array}{c}\left\langle\mathcal{B}_{z}\right\rangle \\
{[\mathrm{G}]}\end{array}$ & $\begin{array}{c}\sigma_{z} \\
{[\mathrm{G}]}\end{array}$ & MJD & $\begin{array}{c}\left\langle\mathcal{B}_{z}\right\rangle \\
{[\mathrm{G}]}\end{array}$ & $\begin{array}{c}\sigma_{z} \\
{[\mathrm{G}]}\end{array}$ \\
\hline 52531.108661 & -701 & 79 & 52531.222581 & -1123 & 82 \\
\hline 52531.112774 & -822 & 79 & 52531.224908 & -937 & 107 \\
\hline 52531.117013 & -862 & 70 & 52531.241321 & -468 & 76 \\
\hline 52531.12 & -765 & 70 & 525 & -695 & 76 \\
\hline 525 & -789 & 70 & 525 & -588 & 79 \\
\hline 525 & -808 & 70 & 525 & -696 & 79 \\
\hline 5 & -574 & 70 & 525 & -672 & 85 \\
\hline 525 & -709 & 73 & 525 & -717 & 85 \\
\hline 5253 & -640 & 70 & 52531. & -425 & 79 \\
\hline 52531.13 & -689 & 73 & 52531.2 & -734 & 76 \\
\hline 52531.137511 & -895 & 88 & 52531.259126 & -676 & 76 \\
\hline 52531.14 & -696 & 79 & 52531.261359 & -651 & 79 \\
\hline 52531 & -655 & 70 & 52531 & -621 & 79 \\
\hline 52 & -728 & 73 & 52 & -705 & 79 \\
\hline 5 & -747 & 92 & 525 & -655 & 82 \\
\hline 525 & -763 & 88 & 525 & -629 & 76 \\
\hline 5 & -682 & 79 & 525 & -586 & 82 \\
\hline 525 & -764 & 73 & 52 & -700 & 82 \\
\hline 52531 & -629 & 79 & 5253 & -718 & 79 \\
\hline 52531.158861 & -708 & 73 & 52531.280145 & -661 & 79 \\
\hline 52531.1 & -699 & 73 & 52531. & -587 & 82 \\
\hline 525 & -512 & 70 & 525 & -758 & 85 \\
\hline 525 & -579 & 70 & 525 & -626 & 82 \\
\hline 525 & -671 & 76 & 525 & -784 & 82 \\
\hline & -553 & 76 & 55 & -698 & 82 \\
\hline & -596 & 76 & 5 & -563 & 85 \\
\hline 52531.17 & -746 & 73 & 52531.2 & -638 & 85 \\
\hline 5253 & -812 & 70 & 52531 & -756 & 85 \\
\hline 52531. & -742 & 70 & 5253 & -533 & 85 \\
\hline 52531.182705 & -727 & 70 & 52531.302620 & -662 & 85 \\
\hline 52531.184983 & -712 & 70 & 52531.304873 & -759 & 85 \\
\hline 52531.187294 & -702 & 63 & 52531.307128 & -656 & 92 \\
\hline 52531.1 & -661 & 66 & 52531.309384 & -535 & 88 \\
\hline 52531.191868 & -795 & 70 & 52531.311642 & -547 & 88 \\
\hline 52531.194151 & -686 & 70 & 52531.314797 & -407 & 85 \\
\hline & -567 & 63 & 52531 & -595 & 88 \\
\hline 525 & -695 & 73 & 52531 . & -945 & 98 \\
\hline 52531.201013 & -744 & 73 & 52531. & -632 & 92 \\
\hline 52531.203297 & -655 & 70 & 52531.323855 & -728 & 98 \\
\hline 52531.205589 & -681 & 66 & 52531.332912 & -735 & 111 \\
\hline 52531.207877 & -691 & 66 & 52531.335189 & -733 & 98 \\
\hline 52531.210168 & -694 & 66 & 52531.337471 & -767 & 98 \\
\hline 52531.212455 & -768 & 70 & 52531.339747 & -827 & 101 \\
\hline 52531.215691 & -758 & 70 & 52531.342024 & -556 & 98 \\
\hline 52531.217987 & -592 & 70 & 52531.344306 & -614 & 98 \\
\hline 52531.220277 & -424 & 73 & & & \\
\hline
\end{tabular}




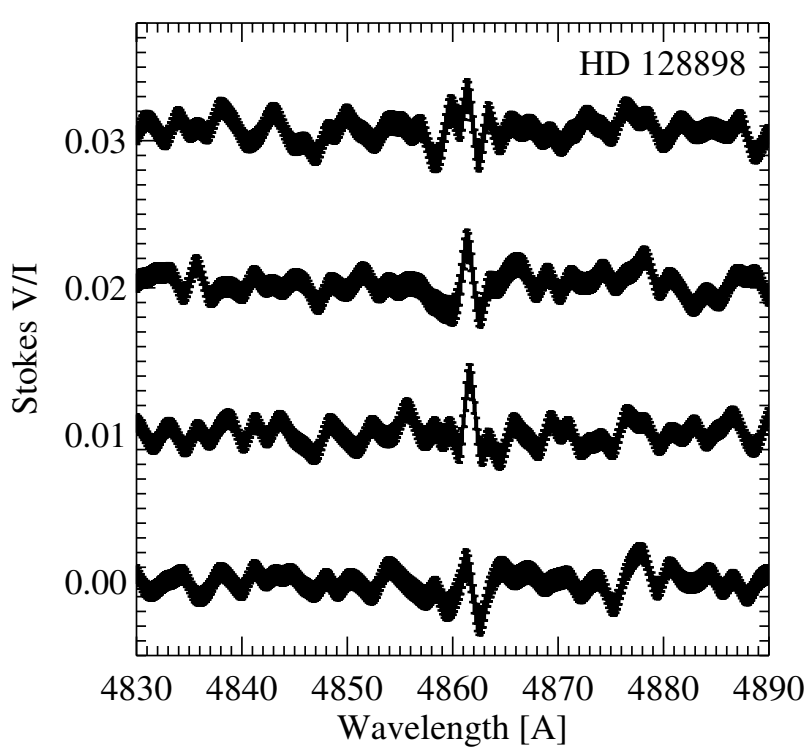

Fig. 2. Pulsational behaviour of the Stokes $V$ profiles in the region of the $\mathrm{H}_{\beta}$ line over four consecutive exposures in the star HD 128898 with the smallest measured longitudinal magnetic field. As in Fig. 1, the thickness of the plotted lines corresponds to the uncertainty of the measurements of polarization determined from photon noise.

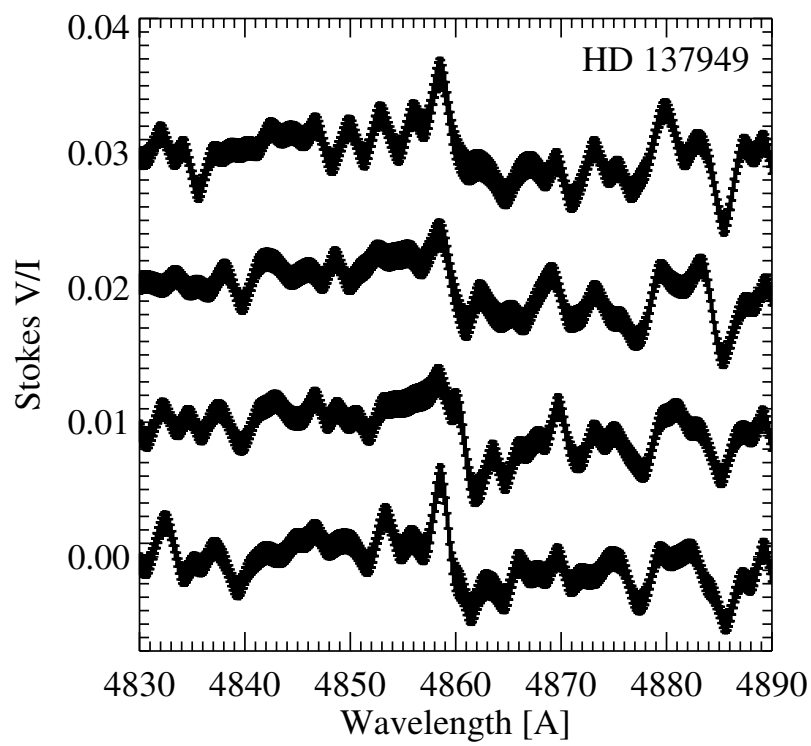

Fig. 3. Pulsational behaviour of the Stokes $V$ profiles in the region of the $\mathrm{H}_{\beta}$ line over four consecutive exposures in the star HD 137949 with the largest measured longitudinal magnetic field. As in Fig. 1, the thickness of the plotted lines corresponds to the uncertainty of the measurements of polarization determined from photon noise.

other Balmer lines, a Fourier analysis has been done using different sets of magnetic field determinations including those in the full wavelength range and the measurements within the wavelength windows around the individual Balmer lines $\mathrm{H}_{\alpha}$, $\mathrm{H}_{\beta}, \mathrm{H}_{\gamma}$ and $\mathrm{H}_{\delta}$. Shortwards from $\mathrm{H}_{\delta}$ the measurements of the magnetic field using hydrogen lines show large uncertainties, up to $1000 \mathrm{G}$, due to a lower photon count rate and lower Zeeman splitting in the blue region.

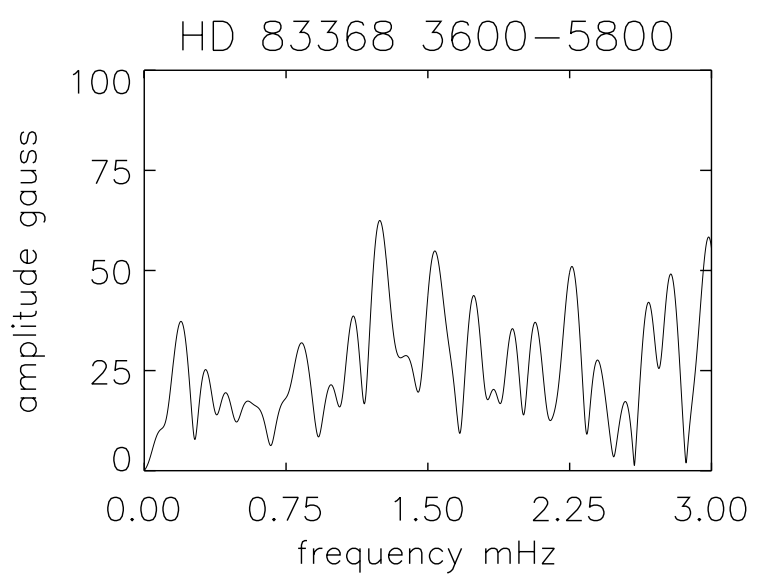

Fig. 4. An amplitude spectrum of the magnetic field measurements of HD 83368 over the entire wavelength range studied. This is typical of the amplitude spectra for the stars in the sample. The highest peak is at $v=1.246 \mathrm{mHz}(P=13.38 \mathrm{~min})$ which is not at the known pulsation frequency of $1.428 \mathrm{mHz}(P=11.67 \mathrm{~min})$, hence is probably a noise peak.

In the following we present the results of Fourier analyses of magnetic field measurements over several pulsation cycles for each star in the sample. Least squares fits have been applied to determine the amplitude spectrum of each star.

HD 83368 (HR 3831) is singly periodic with a pulsation period of $11.67 \mathrm{~min}$ (Kurtz 1982). We have obtained 60 series of magnetic field measurements with exposure times of 10-18 s over 14 consecutive pulsation cycles. Taking into account the overheads one magnetic field measurement took $\approx 2.5 \mathrm{~min}$. In Fig. 4 we show the amplitude spectrum of HD 83368 obtained from the measurements in the whole spectral region from 3600 to $5800 \AA$. The highest peak at $1.246 \mathrm{mHz}$ for the measurements in the whole spectral region is not at the known photometric pulsation frequency $(1.428 \mathrm{mHz})$. None of the amplitude spectra for the measurements of Balmer lines shows a signal at the known photometric pulsation frequency either. It seems highly unlikely that a pulsation frequency previously undetected in photometric observations, may actually be present in HD 83368, which is one of the best studied roAp stars. The standard deviation of the measurements is $108 \mathrm{G}$ - significantly higher than the formal errors given in Table 2. This suggests either that there is a signal buried in the noise, or that the formal error estimates are too low.

HD 101065 (Przybylski's star) pulsates with one principal mode with a frequency of $v=1.373 \mathrm{mHz}(P=12.14 \mathrm{~min})$ and with two other much lower amplitude frequencies (Martinez \& Kurtz 1990). This star has the highest photometric pulsation amplitude $\left(\Delta B_{\max }=13\right.$ mmag peak-to-peak $)$ among the stars in our sample, and was at first observed for 12 consecutive pulsation cycles in April 2002. Thirty series with exposure times of 60-80 s were acquired and about 4.5 min were spent for a single measurement of the magnetic field. The highest peak in the amplitude spectrum (Fig. 5, upper panel) is at a frequency of $1.365 \mathrm{mHz}$ with an amplitude of $39 \pm 12 \mathrm{G}$. This $3.2 \sigma$ signal is at the known photometric pulsation frequency, hence is possibly real. The False Alarm Probability of a $3.2 \sigma$ peak 


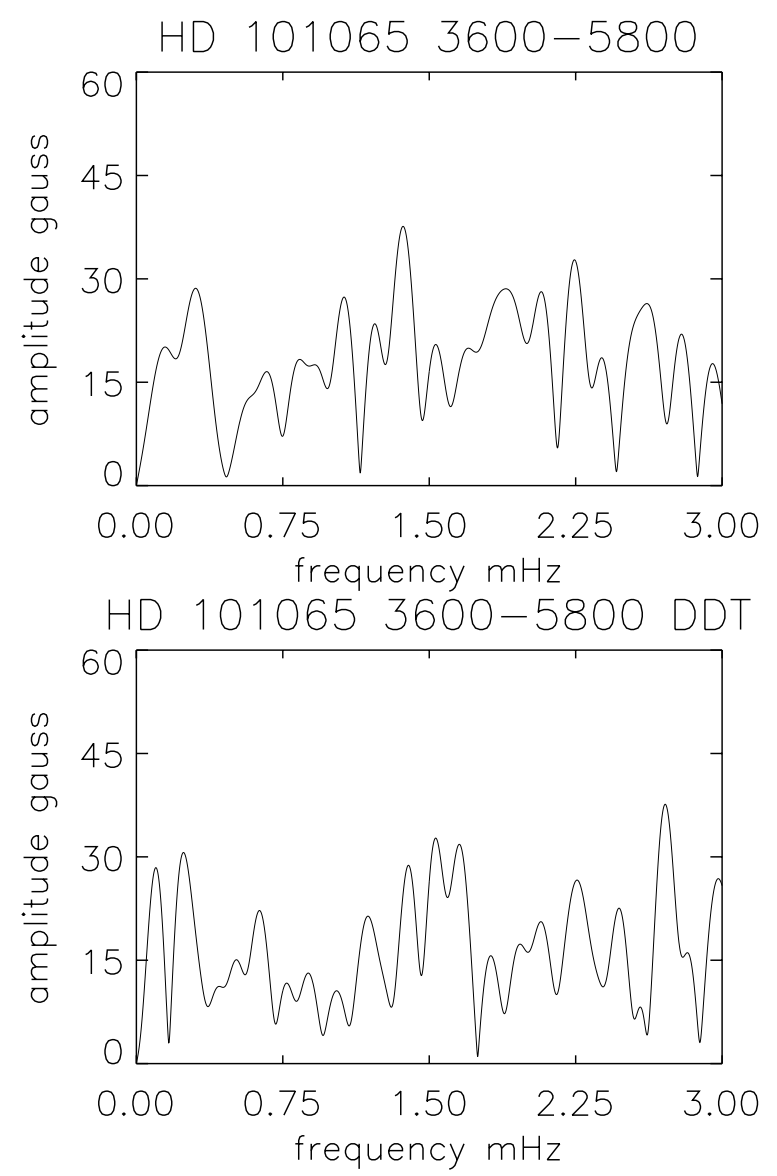

Fig. 5. Amplitude spectra of the magnetic field measurements of HD 101065. The top panel shows the amplitude spectrum for the first $2.4 \mathrm{~h}$ run with the highest peak at the known photometric pulsation frequency, $1.365 \mathrm{mHz}$, with an amplitude of $39 \pm 12 \mathrm{G}$. The lower panel shows the amplitude spectrum of a $4 \mathrm{~h}$ run under Director's Discretionary Time that fails to confirm the highest peak in the upper panel.

somewhere in the amplitude spectrum is high, since many independent frequencies have been searched. But the False Alarm Probability at a particular frequency, namely the known pulsation frequency, is much lower.

The theoretical considerations of pulsationally-modulated magnetic field variations in case of the star HD 101065 show that the pulsation could give rise to magnetic field variations up to $10^{2} \mathrm{G}$ if we assume the radial velocity amplitude to be of the order of $1 \mathrm{~km} \mathrm{~s}^{-1}:\left|\delta B_{\theta} / B\right| \sim|\partial \xi / \partial r| \sim P \delta V /(2 h \pi) \sim 0.1$, hence $\delta B_{\theta} \approx 100 \mathrm{G}$, in this case, consistent with this possible detection. However, we should note that no study of radial velocity variations over the pulsation cycle has ever been carried out for HD 101065. With this encouraging indication four additional hours with FORS1 were granted by the Director's Discretionary Time Committee (Programme No. 270.D-5023) to test our possible detection. The 72 additional measurements were obtained in March 2003; they are presented in Table 8. Unfortunately, we did not confirm the result, as can be seen in the lower panel of Fig. 5. The new observations do not rule out the possibility of variation of the magnetic field with the known photometric frequency, but they also certainly do not confirm it.
Table 8. Mean longitudinal magnetic field for a four hours time series of HD 101065 obtained in March 2003.

\begin{tabular}{ccc|ccc}
\hline \hline MJD & $\left\langle\mathcal{B}_{z}\right\rangle$ & $\sigma_{z}$ & MJD & $\left\langle\mathcal{B}_{z}\right\rangle$ & $\sigma_{z}$ \\
& {$[\mathrm{G}]$} & {$[\mathrm{G}]$} & & {$[\mathrm{G}]$} & {$[\mathrm{G}]$} \\
\hline 52701.169601 & -1031 & 50 & 52701.254376 & -1121 & 59 \\
52701.172534 & -973 & 49 & 52701.256476 & -1081 & 60 \\
52701.175441 & -910 & 50 & 52701.258483 & -1073 & 62 \\
52701.178343 & -987 & 49 & 52701.260479 & -1095 & 67 \\
52701.181240 & -982 & 49 & 52701.262477 & -986 & 66 \\
52701.184140 & -964 & 48 & 52701.264478 & -1036 & 62 \\
52701.187049 & -954 & 48 & 52701.266475 & -1019 & 64 \\
52701.189827 & -894 & 52 & 52701.268472 & -925 & 66 \\
52701.192397 & -1072 & 53 & 52701.270472 & -1005 & 64 \\
52701.194950 & -982 & 53 & 52701.272471 & -950 & 65 \\
52701.197503 & -889 & 51 & 52701.274468 & -1081 & 63 \\
52701.200059 & -952 & 51 & 52701.276471 & -958 & 68 \\
52701.202527 & -835 & 59 & 52701.278473 & -1046 & 74 \\
52701.204782 & -792 & 59 & 52701.280492 & -1062 & 65 \\
52701.207092 & -1007 & 56 & 52701.282594 & -1115 & 62 \\
52701.209416 & -981 & 55 & 52701.284715 & -974 & 61 \\
52701.211743 & -905 & 54 & 52701.286833 & -1041 & 62 \\
52701.214011 & -1034 & 57 & 52701.288956 & -1002 & 59 \\
52701.216236 & -1126 & 57 & 52701.291079 & -1080 & 59 \\
52701.218449 & -949 & 56 & 52701.293201 & -1097 & 62 \\
52701.220655 & -887 & 56 & 52701.295327 & -1076 & 60 \\
52701.222810 & -1135 & 63 & 52701.297456 & -960 & 61 \\
52701.224909 & -1033 & 58 & 52701.299584 & -1077 & 60 \\
52701.227011 & -896 & 60 & 52701.301712 & -963 & 62 \\
52701.229111 & -932 & 58 & 52701.303843 & -936 & 59 \\
52701.231212 & -1076 & 59 & 52701.305974 & -1134 & 60 \\
52701.233315 & -987 & 60 & 52701.308101 & -972 & 60 \\
52701.235415 & -1006 & 56 & 52701.310327 & -995 & 58 \\
52701.237517 & -1076 & 59 & 52701.312510 & -1036 & 70 \\
52701.239618 & -1081 & 58 & 52701.314551 & -895 & 67 \\
52701.241720 & -985 & 60 & 52701.316673 & -1061 & 61 \\
52701.243827 & -1072 & 61 & 52701.318811 & -837 & 63 \\
52701.245936 & -1063 & 60 & 52701.320952 & -1064 & 61 \\
52701.248043 & -1006 & 60 & 52701.323091 & -940 & 60 \\
5250150 & -1024 & 58 & 52701.325230 & -1014 & 68 \\
525256 & -1090 & 58 & 52701.327429 & -991 & 58 \\
\hline 5 & & & & &
\end{tabular}

HD 128898 ( $\alpha$ Cir; HR 5463) is the brightest roAp star at $V=3.2$. It pulsates with one dominant mode with a frequency of $v=2.44 \mathrm{mHz}(P=6.8 \mathrm{~min})$, and several much lower amplitude frequencies. The principal mode is rotationally modulated by a very small amount with the rotation period of 4.463 day (Kurtz et al. 1994). We obtained 26 series of magnetic measurements with exposure times of 3 to $4 \mathrm{~s}$ distributed 
Table 9. Rapidly oscillating Ap stars studied for magnetic field pulsations.

\begin{tabular}{|c|c|c|c|c|c|c|c|c|}
\hline Star & $\begin{array}{c}T_{\text {eff }} \\
{[\mathrm{K}]}\end{array}$ & $\log g$ & $\begin{array}{c}v \sin i \\
{\left[\mathrm{~km} \mathrm{~s}^{-1}\right]}\end{array}$ & $\begin{array}{l}P_{\text {rot }} \\
{[\mathrm{d}]}\end{array}$ & $\begin{array}{c}\left\langle\mathcal{B}_{z}\right\rangle_{\text {aver }} \\
{[\mathrm{G}]}\end{array}$ & $\begin{array}{c}\sigma_{z \text {,aver }} \\
{[\mathrm{G}]}\end{array}$ & $\begin{array}{c}P_{\text {puls }} \\
{[\mathrm{min}]}\end{array}$ & $\begin{array}{r}\Delta B_{\max } \\
{[\mathrm{mmag}]}\end{array}$ \\
\hline HD $83368^{1}$ & 7960 & 4.0 & 33.0 & 2.85 & $-847 \pm 108$ & 56 & 11.6 & 10 \\
\hline HD $101065^{2}$ & 6600 & 4.2 & 3.5 & & $-1014 \pm 72$ & 57 & 12.1 & 13 \\
\hline HD $128898^{1}$ & 7998 & 4.2 & 13.0 & 4.48 & $-239 \pm 136$ & 62 & 6.8 & 5 \\
\hline HD $137949^{1}$ & 7030 & 3.9 & & $>75 y$ ? & $2146 \pm 55$ & 51 & 8.3 & 3 \\
\hline HD $201601^{1}$ & 7620 & 4.0 & & $>70 y$ & $-1072 \pm 173$ & 55 & 12.4 & 3 \\
\hline HD $217522^{3}$ & 6808 & 4.0 & 3.0 & & $-686 \pm 111$ & 79 & 13.9 & 4 \\
\hline
\end{tabular}

${ }^{1}$ Hubrig et al. (2000); ${ }^{2}$ Cowley et al. (2000); ${ }^{3}$ Hubrig et al. (2002).

over 9 consecutive pulsation cycles. The highest peak found in the amplitude spectrum $(v=2.784 \mathrm{mHz} ; A=104 \mathrm{G})$ from the measurements obtained in the full wavelength range is not at the known photometric pulsation frequency $(2.442 \mathrm{mHz})$. No significant peaks were found in the amplitude spectra from the measurements of the individual Balmer lines.

HD 137949 (33 Lib) pulsates in one mode with a frequency of $v=2.01 \mathrm{mHz}(P=8.3 \mathrm{~min})$ (Kurtz 1991). This star exhibits the strongest longitudinal magnetic field among the stars of our sample. We obtained 30 series of magnetic measurements with exposure times of $30 \mathrm{~s}$ over 10 pulsation cycles. No peak at the known photometric frequency $(2.014 \mathrm{mHz})$ was found in the amplitude spectra of the magnetic field measurements obtained in the full wavelength range; the highest noise peaks had amplitudes of only $30 \mathrm{G}$. No significant peaks were found in the amplitude spectra from the measurements of the individual Balmer lines.

HD 201601 ( $\gamma$ Equ; HR 8097) is the second brightest roAp star at $V=4.7$. It pulsates with four frequencies with periods near 12.3 min (Martinez et al. 1996). Magnetic measurement data were taken sporadically because of clouds passing during the observations. We obtained in all only 18 series with exposure times of 2 to $5 \mathrm{~s}$. No signal was detected, and, as a consequence of the sparseness of the data, the highest noise peaks in the amplitude spectra are at $170 \mathrm{G}$ - far higher than in our data sets for other stars. No significant peaks were found in the amplitude spectra from the measurements of the individual Balmer lines.

HD 217522 (CPD $\left.-45^{\circ} 10378\right)$ was originally discovered to pulsate in a single mode with a period of $13.7 \mathrm{~min}$ (Kurtz 1983). Further observations by Kreidl et al. (1991) found another pulsation mode with a period of 8.3 min that was not present in the discovery data set, indicating transient modes, or strong amplitude modulation. Magnetic measurements were taken over 24 consecutive cycles (of the original mode) with 91 series with the exposure time of $30 \mathrm{~s}$. We found in the amplitude spectrum obtained from the measurements in the full wavelength range a highest peak with an amplitude of $36 \mathrm{G}$ at $1.673 \mathrm{mHz}$ corresponding to a period of $9.97 \mathrm{~min}$. This peak is not significant and does not match any known photometric pulsation period. No significant peaks were found in the amplitude spectra from the measurements of the individual Balmer lines.

\section{Discussion}

The basic data of our sample of roAp stars are listed, star by star, in Table 9. Successive columns give the HD number, the effective temperature, the gravity, the value of $v \sin i$ and of the rotation period, if known. The mean value, for every star, of all the longitudinal field measurements of Tables 2 to 8 , $\left\langle\mathcal{B}_{z}\right\rangle_{\text {aver }}$, and the standard deviation of the individual measurements about this mean, appears in Col. 6. The mean of all the standard deviations of the individual measurements, $\sigma_{z \text {,aver }}$ from the same tables, is presented in Col. 7. In Cols. 8 and 9 we list the photometric pulsation period $P_{\text {puls }}$ and the photometric pulsation amplitude $\Delta B_{\max }$. The indexes 1-3 in the HD number column refer to the literature sources of the atmospheric parameters.

With UT3 of the VLT and FORS 1, we measured the mean longitudinal field variation over the pulsation cycle in six roAp stars to begin the study of how the magnetic field and pulsation interact. Only the star HD 101065, which has one of the highest photometric pulsation amplitudes, showed a potential signal at the known photometric pulsation frequency at the $3 \sigma$ level. We found a signal for magnetic variability with a frequency of $1.365 \mathrm{mHz}$ and an amplitude of $39 \pm 12 \mathrm{G}$ in this star. First theoretical considerations led to the result that in case of the star HD 101065 the pulsation could give rise to magnetic field variations $\left|\frac{\delta B_{\theta}}{B}\right| \sim 0.1 \rightarrow\left|\delta B_{\theta}\right| \approx 100 \mathrm{G}$, consistent with our possible detection. However, our second attempt to measure magnetic variability in this star during $4 \mathrm{~h}$ failed to obtain a positive detection again. Given the noise level, the amplitude spectrum of the new observations does not rule out the possibility of variations at the level found from the measurements in April, but it certainly does not confirm them.

Our study has come close to the limits of what is currently feasible for the measurements of magnetic fields in roAp stars with FORS 1 at the VLT. Although the low resolution spectropolarimetry of hydrogen Balmer lines obtained with FORS1 represents a powerful diagnostic tool for detection of stellar magnetic fields, the accuracy of spectropolarimetric measurements with FORS 1 has not yet been quite high enough to detect magnetic pulsation variations of the order of a few tens of $\mathrm{G}$ that we expect to be present. Given our possible marginal detection with a few hours of observations of HD 101065, an $8 \mathrm{~h}$ run over about 40 pulsation cycles should 
increase our signal-to-noise ratio by nearly a factor of two and show a clear result.

Because of the low resolution of the FORS 1 spectra, no magnetic field measurements can be carried out using exclusively doubly-ionized rare-earth lines which show the largest pulsational radial velocity amplitudes in roAp stars. In general, pulsational amplitudes found in the hydrogen lines are lower than for doubly-ionized rare-earth lines implying a lower level of the expected magnetic field variations. The advantage of using high-resolution spectropolarimetry has been demonstrated in the very recently published study of magnetic field variations over the pulsation period in the roAp star $\gamma$ Equ by Leone $\&$ Kurtz (2003). A series of spectra has been obtained on the $3.55 \mathrm{~m}$ Telescopio Nazionale Galileo (TNG) with the high resolution spectrograph SARG equipped with a polarimeter. Magnetic field measurements of four strong lines of Nd III showed a variability over the pulsation cycle with an amplitude in the range $112-240 \mathrm{G}$.

As mentioned in the introduction, the primary purpose of our work was to obtain observational information on magnetic field variations and its very interest was to provide constraints for further development of theoretical models. In this respect, it is noteworthy that adopting the measured radial velocity amplitude of the Nd III lines of the order of $400 \mathrm{~m} \mathrm{~s}^{-1}$, the expected magnitude of magnetic field variations for $\gamma$ Equ according to the theoretical estimate of Sect. 1 is at least two times lower than the magnitude observed by Leone \& Kurtz.

To summarize, from the observational point of view there is a need to observe more roAp stars in order to understand how the pulsation pattern interacts with the magnetic field on the time-scale of the pulsations. The approach should consist in achieving better accuracy of magnetic field measurements taking advantage of the large light collecting power of $4 \mathrm{~m}$ to $8 \mathrm{~m}$ class telescopes and high-resolution spectropolarimeters like SARG on the $3.55 \mathrm{~m}$ TNG telescope or ESPaDOnS which will be installed onto the Canada-France-Hawaii telescope this year. In the absence of spectropolarimetric instruments on large telescopes, this study can be complemented by high spectral resolution, high time resolution spectra of roAp stars with magnetically split lines obtained in unpolarized light at $4 \mathrm{~m}$ to $8 \mathrm{~m}$ class telescopes. Such observations, which are sensitive to the mean magnetic field modulus rather than the mean longitudinal magnetic field considered here, can potentially detect lower amplitude variations of this field moment (uncertainties of individual measurements based on a single line can be as low as $\sim 25 \mathrm{G}$ ). Observations of this type using the VLT have already been carried out for one roAp star (Mathys et al., in preparation).

\section{References}

Appenzeller, I., Fricke, K., Furtig, W., et al. 1998, The Messenger, 94, 1

Bagnulo, S., Szeifert, T., Wade, G. A., Landstreet, J. D., \& Mathys, G. 2002, A\&A, 356, 218

Baldry, I. K., \& Bedding, T. R. 2000, MNRAS, 318, 341

Baldry, I. K., Viskum, M., Bedding, T. R., Kjeldsen, H., \& Frandsen, S. 1999, MNRAS, 302, 381

Balona, L. A. 2002, MNRAS, 337, 1059

Balona, L. A., \& Zima, W. 2002, MNRAS, 336, 873

Bigot, L., \& Dziembowski, W. A. 2002, A\&A, 391, 235

Bigot, L., Provost, J., Berthomieu, G., Dziembowski, W. A., \& Goode, P. 2000, A\&A, 356, 218

Cowley, C. R., Ryabchikova, T., Kupka, F., et al. 2000, MNRAS, 317, 29

Cunha, M. S., \& Gough, D. 2000, MNRAS, 319, 102

Handler, G., Weiss, W. W., Paunzen, P., et al. 2002, MNRAS, 330, 153

Hatzes, A. P., Kanaan, A., \& Mkrtichian, D. 1999, ASP Conf. Ser., 185,183

Hubrig, S. North, P., \& Mathys, G. 2000, ApJ 539, 352

Hubrig, S., Cowley, C. R., Bagnulo, S., et al. 2002, ASP Conf. Ser., 279,365

Hubrig, S., Bagnulo, S., Kurtz, D. W., et al. 2003, ASP Conf. Ser., in press

Hubrig, S., Szeifert, T., Schöller, M., Mathys, G., \& Kurtz, D. W. 2004, A\&A, 415, 685

Knudsen, D. 2000, MSc Thesis

Kochukhov, O., \& Ryabchikova, T. 2001, A\&A, 374, 615

Kochukhov, O., \& Ryabchikova, T. 2001, A\&A, 377, L22

Kreidl, T. J., Kurtz, D. W., Bus, S. J., et al. 1991, MNRAS, 250, 477

Kurtz, D. W. 1982, MNRAS, 200, 807

Kurtz, D. W. 1983, MNRAS, 205, 3

Kurtz, D. W. 1990, ARA\&A, 28, 607

Kurtz, D. W. 1991, MNRAS, 249, 468

Kurtz, D. W., Elkin, V., \& Mathys, G. 2003, MNRAS, in press

Kurtz, D. W., \& Martinez, P. 2000, Baltic Astron., 9, 253

Kurtz, D. W., Sullivan, D. J., Martinez, P., \& Tripe, P. 1994, MNRAS, 270,674

Landstreet, J. D. 1982, ApJ, 258, 639

Leone, F., \& Kurtz, D. W. 2003, A\&A, 407, L67

Martinez, P., \& Kurtz, D. W. 1990, MNRAS, 242, 636

Martinez, P., Weiss, W. W., Nelson, M. J., et al. 1996, MNRAS, 282, 243

Mathys, G. 2003, ASP Conf. Ser., 216, in press

Mathys, G., Hubrig, S., Landstreet, J. D., Lanz, T., \& Manfroid, J. 1997, A\&AS, 123, 353

Mathys, G., Manfroid, J., \& Wenderoth, E. 2003, in preparation

Matthews, J. M. 1991, PASP, 103, 5 